\title{
Clinical Correlation between Muscle Damage and Oswestry Disability Index Score after Open Lumbar Surgery: Does Open Surgery Reduces Functional Ability?
}

\author{
Rushama Tandon, Vivek Kiyawat, Neeraj Kumar \\ Department of Orthopedic Surgery, Northern Railway Central Hospital, New Delhi, India
}

\begin{abstract}
Study Design: Single-surgeon, single-center prospective study with prospective data collection.
Purpose: To clinically evaluate muscle damage after open lumbar surgery and its relationship to functional activity and to validate the improvement in function as indicated by improved Oswestry Disability Index (ODI) score despite muscle damage.

Overview of Literature: Few studies have analyzed the functional loss and recovery pattern of muscles after open lumbar surgery. Methods: The study included 30 patients who underwent open lumbar spine fusion surgery at our institution between August 2013 and May 2015. Preoperatively and at 6 months postoperatively, the patients were subjected to functional, biochemical, electrophysiological, and radiological assessments as outpatients, and the results were compared.

Results: Mean preoperative and 6-month postoperative values were as follows: creatine phosphokinase levels, 133.07 \pm 17.57 and $139 \pm 17.7 \mathrm{U} / \mathrm{L}(p<0.001)$; Visual Analog Scale scores for backache, $6.73 \pm 0.88$ and $3.27 \pm 0.96(p<0.001)$; and ODI scores, $41.6 \pm 5.51$ and $22.4 \pm 4.48(p<0.001)$, respectively. Preoperatively, electrophysiological studies showed that $20 \%$ of the patients had a polyphasic configuration whereas at 6 months postoperatively, all patients had polyphasic configuration $(p<0.001)$. The mean cross-sectional area of the multifidus observed using magnetic resonance imaging (MRI) decreased from $742.67 \pm 76.62 \mathrm{~mm}^{2}$ preoperatively to $598.27 \pm 66.38$ $\mathrm{mm}^{2} 6$ months postoperatively $(p<0.001)$, with all the patients exhibiting grade 2 atrophy.

Conclusions: Open lumbar fusion surgery resulted in significant damage to the lumbar paraspinal muscles, as indicated by a reduction in the cross-sectional area of the multifidus by MRI and denervation of the multifidus demonstrated using electromyography. Nevertheless, the patients reported reduced back pain and improved quality of life, which may have been due to increased stability of the previously unstable lumbar spinal segment after the surgery.
\end{abstract}

Keywords: Lumbar spine; Open surgery; Functional disability; Muscle damage

\section{Introduction}

Lumbar surgery is a major surgical option for the treat- ment of lumbar spinal disease. However, many studies have reported mechanical and physiological changes after the surgery, which can result in undesirable problems,

Received Jun 11, 2017; Revised Oct 2, 2017; Accepted Oct 10, 2017

Corresponding author: Rushama Tandon

Department of Orthopedic Surgery, Northern Railway Central Hospital, Basant Lane, Connaught Place, New Delhi 110055, India

Tel: +91-9717630535, E-mail: rushtan@rediffmail.com 
such as spinal stiffness, acceleration of adjacent segment degeneration, and chronic low back pain [1]. Reported changes in the paraspinal muscles after surgery include a loss of muscle thickness, edematous and fatty changes observed in magnetic resonance imaging (MRI), and lower muscle potential in electromyography (EMG). Pseudoarthrosis, low back pain, spinal stiffness, and instability have also been reported following spinal fusion procedures [25].

Lumbar fusion eliminates the motion of functional spinal segments, and this may overload adjacent segments. Furthermore, surgical trauma and disuse of the immobile segments can decrease the volume of the paraspinal muscles. Multiple studies have addressed the issue of postoperative changes to the paraspinal muscles with a majority of these studies concluding that lumbar fusion adversely affects the back muscles and reduces muscle mass, regardless of the surgical approach used [6-8].

The multifidus muscle is the most important muscle for lumbar segmental stability. It is the largest of the paraspinal muscles and is the closest of them to the spine. A recent study with an architectural design demonstrated that the multifidus muscle was uniquely designed to act as a stabilizer and to produce large forces [9]. The multifidus muscle is only innervated by the medial branches of the dorsal ramus with no intersegmental nerve supply [10], and it is the muscle that is most directly affected by heat injury during retraction and dissection from the adjacent spine. To prevent multifidus muscle injury during surgery and other surgical approaches, an intermittent release of the retractor has been recommended [11]. Suwa et al. [12] concluded that the Wiltse paraspinal approach to the lumbar spine allows manual dissection of the multifidus and longissimus muscles to expose the transverse process and lateral aspect of the facet joint with minimal retraction; thus, this approach may reduce the risk of heat and retraction injuries to the paraspinal muscles. This approach, along with the percutaneous fixation technique, is known to preserve paraspinal muscles better in patients with fusion surgery, allowing severe muscle injuries and paraspinal muscle denervation to be avoided [13].

Remes et al. [14] noted that in the long term, all fusion procedures cause damage to back muscles. They found that anterior lumbar interbody fusion, which does not involve direct manipulation of the back muscles, was associated with back muscle atrophy as observed in posterior lumbar interbody fusion and circumferential fusion. This implies that loss of motion affects the paraspinal muscle integrity regardless of the surgical approach used, especially in the long term. Thus, motion preservation may play an important role in the maintenance of back muscle integrity, besides initial surgical trauma [14].

The aim of the present study was to evaluate the extent of muscle change after open lumbar spine fusion surgery, and the clinical relevance of the change in terms of functional disability.

\section{Materials and Methods}

This prospective study included patients aged $\geq 18$ years that underwent open lumbar spine fusion surgery at our institution between August 2013 and May 2015. The exclusion criteria were previous surgery for spinal disease, neuromuscular disorder, spinal malignancy, spinal infection, or disagreement for participation. In total, 30 patients were included. The indications for open posterior lumbar spinal fusion included isthmic spondylolisthesis, unstable spinal stenosis syndromes (degenerative spondylolisthesis or degenerative scoliosis), a traumatic unstable fracture, and objective segmental instability. All the patients underwent following functional, biochemical, electrophysiological, and radiological evaluations as outpatients, both preoperatively and 6 months postoperatively.

\section{Biochemical evaluation}

Serum creatine phosphokinase (CPK) concentrations were measured.

\section{Clinical and functional evaluation}

The patients rated the intensity of their low back pain using a 0-10 Visual Analog Scale (VAS), with 0 being "no pain" to 10 being "the worst pain imaginable." Functional improvement was evaluated by using the ODI questionnaire and muscle charting.

\section{Electrophysiological evaluation}

EMG studies were used to evaluate insertional activity, spontaneous activity (fibrillation and positive sharp waves), and motor unit potential (amplitude, duration, and configuration) in the lumbar paraspinal muscles. 


\section{Radiological evaluation}

Plain radiography and standard sagittal and axial T1- and T2-weighted MRI scans of the lumbar spine were acquired preoperatively and 6 months postoperatively. A single radiologist, blinded to the allocated treatment, measured the mean cross-sectional area of the multifidus muscle bulk at the level of the surgery on T2-weighted axial MRI. Atrophy was graded based on the percentage replacement of multifidus muscle bulk with fat and fibrous tissue, as follows: grade 0 (normal), grade 1 (mild, with $<10 \%$ replacement), grade 2 (moderate, with 10\%-50\% replacement), and grade 3 (severe, with $>50 \%$ replacement).

\section{Statistical analysis}

Statistical analysis was performed using SPSS ver. 17.0 (SPSS Inc., Chicago, IL, USA), with $p<0.05$ considered statistically significant.

\section{Results}

A total of 30 patients (28 men and two women; mean age, 53.8 years) were enrolled in the study. All the patients presented with back pain; this was associated with radiculopathy in 24 cases, weakness of the lower limbs in 20 cases, and bowel bladder involvement in two cases. The vertebral involvement was 1-level in six cases; 2-level in 20 cases, and 3-level in four cases. The mean duration of surgery was 95.3 minutes. A 2-level fusion was performed in 16 cases and 3-level fusion in the other 14 cases.

The mean CPK concentration changed from $133.07 \pm 17.57 \mathrm{U} / \mathrm{L}$ preoperatively to $139 \pm 17.7 \mathrm{U} / \mathrm{L}$ at 6 months postoperatively $(p<0.001)$. The mean VAS score for backache changed from $6.73 \pm 0.88$ preoperatively to $3.27 \pm 0.966$ months postoperatively $(p<0.001)$, and the mean ODI score changed from $41.6 \pm 5.51$ preoperatively to $22.4 \pm 4.486$ months postoperatively $(p<0.001)$ (Table 1$)$.

\section{Electrophysiological evaluation}

Preoperatively, $80 \%$ of the patients presented with a motor unit potential of normal duration, while $20 \%$ showed increased duration; at 6 months postoperatively, all patients showed increased duration. Similarly, the amplitude was normal in $80 \%$ and increased in $20 \%$ of the patients preoperatively, but it increased in all the patients at 6 months postoperatively. Preoperatively, $20 \%$ of the patients showed a polyphasic configuration and the other $80 \%$ presented a normal configuration; all patients showed a polyphasic configuration at 6 months postoperatively $(p<0.001)$ (Table 2).

\section{Radiological evaluation}

The mean cross-sectional area of the multifidus decreased from $742.67 \pm 76.62 \mathrm{~mm}^{2}$ preoperatively to $598.27 \pm 66.38$ $\mathrm{mm}^{2}$ at 6 months postoperatively $(p<0.001)$. Compared

Table 1. Comparison of parameters measured before lumbar fusion surgery and at 6 -month follow-up ( $N=30)$

\begin{tabular}{|c|c|c|c|}
\hline Variable & Preoperative & At 6-month follow-up & $p$-value \\
\hline Mean visual analog scale score for back pain & $6.73 \pm 0.88$ & $3.27 \pm 0.96$ & $<0.001$ \\
\hline Mean Oswestry disability index score for back pain & $41.6 \pm 5.51$ & $22.4 \pm 4.48$ & $<0.001$ \\
\hline Mean creatine phosphokinase level (U/L) & $133.07 \pm 17.57$ & $139 \pm 17.7$ & $<0.001$ \\
\hline Mean cross-sectional area of the multifidus muscle $\left(\mathrm{mm}^{2}\right)$ & $742.67 \pm 76.62$ & $598.27 \pm 66.38$ & $<0.001$ \\
\hline
\end{tabular}

Values are presented as mean \pm standard deviation.

Table 2. Comparison of preoperative and 6-month postoperative electrophysiological evaluation values

\begin{tabular}{lcc} 
Variable & Preoperative & At 6-month follow-up \\
\hline Increased duration of motor unit potential & $6(20)$ & $30(100)$ \\
Increased amplitude & $6(20)$ & $30(100)$ \\
\hline Polyphasic configuration & $6(20)$ & $30(100)$ \\
\hline
\end{tabular}

Values are presented as number (\%). 
with the preoperative values, all the patients exhibited grade 2 atrophy of the multifidus muscle at 6 months postoperatively with radiological fusion evident in 20 cases $(67 \%)$.

\section{Discussion}

Spinal fusion is thought to increase the load at the levels adjacent to the fused segments; together with the underlying natural degeneration, this can lead to adjacent level instability and degeneration $[15,16]$. Multifidus muscle atrophy and fatty infiltration have been observed in MRI studies in patients with lumbar spinal fusion. The conventional posterior open lumbar fusion surgery is also associated with significant trauma to the paraspinal muscles. The long incisions made during the procedure require extensive retraction of muscle from the spinous processes, and the subsequent prolonged wide retraction may result in denervation of the paraspinal musculature [1]. Furthermore, self-retaining retractors cause a significant increase in intramuscular pressure in the erector spinae muscles, which is maintained throughout the surgical procedure. This pressure causes a reduction in capillary perfusion, leading to ischemic changes within the muscle, particularly if the retraction time is longer than 2 hours $[17,18]$. This ischemic damage may be the underlying cause for the electrophysiological and computed tomography abnormalities and overall decrease in trunk muscle strength observed in the paraspinal muscles of patients undergoing the conventional posterior open lumbar fusion surgery [19]. Although the specific impact of muscle retraction on postoperative low back pain and disability is not well established, the extensor muscle dysfunction caused by paraspinal muscle injury during surgery may play an important role in the development of postoperative low back pain [20].

The aim of the present study was to assess the extent of paraspinal muscle change following an open lumbar fusion surgery and to evaluate its clinical relevance. The multifidus muscle is the most directly affected muscle by injury during dissection and retraction; therefore, it was chosen for measurement. We evaluated paraspinal muscles of 30 patients preoperatively and at 6 months postoperatively by measuring CPK concentrations and performing MRI and EMG assessments. The clinical benefit of the surgery was evaluated by VAS and ODI scores. We found that 6 months after lumbar spinal fusion surgery, the mean cross-sectional area of the multifidus had decreased significantly from $742.67 \pm 76.62 \mathrm{~mm}^{2}$ to $598.27 \pm 66.38$ $\mathrm{mm}^{2}(p<0.001)$. This decrease was similar to the findings of several previous studies [21-23]. Additionally, unlike preoperatively, all the patients exhibited grade 2 atrophy of the multifidus muscle at the 6-month follow-up.

Fan et al. [21] measured CPK concentrations one day preoperatively and on days $1,3,5$, and 7 postoperatively. They found that the mean CPK level on postoperative days 1,3 , and 5 was significantly higher in the conventional open surgery group than in the minimally invasive surgery group $(p<0.001)$. In the present study, CPK concentrations increased significantly from $133.07 \pm 17.57 \mathrm{U} /$ L preoperatively to $139 \pm 17.7$ at the 6 -month follow-up $(p<0.001)$.

Hu et al. [24] demonstrated a lower postoperative activity in the paraspinal muscle over the fused segments, which may reflect muscle atrophy. In the present study, EMG showed normal insertional activity in all the patients preoperatively, with all the patients then exhibiting increased insertional activity at 6 months postoperatively $(p<0.001)$. Preoperatively, $80 \%$ and $20 \%$ of the patients presented with grade 0 and grade +1 spontaneous activity, respectively. At 6 months postoperatively, 27\% patients presented with grade $+1,40 \%$ with grade +2 , and $33 \%$ with grade $+3(p<0.001)$ of spontaneous activity. Motor unit potential also significantly increased at 6 months postoperatively $(p<0.001)$. The electrophysiological evaluation demonstrated that open lumbar fusion surgery leads to denervation of the paraspinal muscles.

Fan et al. [21] found that VAS scores for back pain at 6 months after surgery were markedly lower than the preoperative scores $(p<0.001)$. In the present study, the VAS score for low back pain decreased from $6.73 \pm 0.88$ preoperatively to $3.27 \pm 0.96$ at the 6 -month follow-up ( $p<0.001)$. Fan et al. [21] also found that ODI scores at 6 months postoperatively were markedly lower than the preoperative scores $(p<0.001)$. In the present study, the mean ODI score improved from $41.6 \pm 5.51$ to $22.4 \pm 4.48(p<0.001)$. However, the percentage change in ODI scores showed no statistically significant relationship with the percentage change in the cross-sectional area of the multifidus $(p=0.775)$.

Minimally invasive surgery, unlike open spine surgery, is expected to reduce tissue damage, resulting in lesser low back pain, shorter hospitalization and a faster resumption of daily activities. In a comparison of minimally invasive 
and open spine surgery, Cawley et al. [25] found that all 35 analyzed levels demonstrated more pronounced denervation after the open surgery (severity scores, 0.5 versus $1.3 ; p=0.02$ ). Their subanalysis of two-level instrumentations also demonstrated significantly greater denervation in the open surgery group (severity scores, 0.3 versus 1.13; $p=0.04$ ). In addition, a significant difference was noted at non-instrumented adjacent levels between the minimally invasive and open surgery (severity scores, 0.17 versus 1.33 ; $p=0.03$ ). Denervation was noted in $5 / 6$ open surgery levels compared to $1 / 6$ minimally invasive surgery levels. In contrast, Yoo et al. [26] concluded that, after minimally invasive fusion surgery, VAS scores for back pain had not significantly improved ( $p=0.169)$. Moreover, they found no significant difference between preoperative and postoperative ODI scores $(p=0.157)$, and the decrease in the cross-sectional area of the multifidus did not show a statistically significant positive correlation with the ODI scores $(p=0.106)$.

In the present study, there was a significant improvement in the back pain experienced by patients after an open lumbar surgery. The VAS and ODI scores for back pain improved significantly, indicating an improvement in the patients' quality of life.

\section{Conclusions}

Despite a significant damage to the lumbar paraspinal muscles during the open lumbar fusion surgery, as indicated by the denervation of the multifidus using EMG and by a reduction in its cross-sectional area, the patients reported a significant improvement in their back pain and QOL. A review of literature showed that minimally invasive surgery did not necessarily result in greater improvement in functional activity at 6 months postoperatively. The improvements following an open surgery may be due to an increased stability of previously unstable lumbar spinal segments and may be unrelated to the method used. A larger study with more patients and a longer duration of follow-up is necessary to warrant these findings.

\section{Conflict of Interest}

No potential conflict of interest relevant to this article was reported.

\section{References}

1. Fritzell P, Hagg O, Wessberg P, Nordwall A; Swedish Lumbar Spine Study Group. Chronic low back pain and fusion: a comparison of three surgical techniques: a prospective multicenter randomized study from the Swedish lumbar spine study group. Spine (Phila Pa 1976) 2002;27:1131-41.

2. Kim KT, Lee SH, Lee YH, Bae SC, Suk KS. Clinical outcomes of 3 fusion methods through the posterior approach in the lumbar spine. Spine (Phila Pa 1976) 2006;31:1351-7.

3. Helenius I, Remes V, Yrjonen T, et al. Comparison of long-term functional and radiologic outcomes after Harrington instrumentation and spondylodesis in adolescent idiopathic scoliosis: a review of 78 patients. Spine (Phila Pa 1976) 2002;27:176-80.

4. Leong JC, Chun SY, Grange WJ, Fang D. Long-term results of lumbar intervertebral disc prolapse. Spine (Phila Pa 1976) 1983;8:793-9.

5. Luk KD, Lee FB, Leong JC, Hsu LC. The effect on the lumbosacral spine of long spinal fusion for idiopathic scoliosis: a minimum 10-year follow-up. Spine (Phila Pa 1976) 1987;12:996-1000.

6. Kawaguchi Y, Matsui H, Tsuji H. Back muscle injury after posterior lumbar spine surgery: a histologic and enzymatic analysis. Spine (Phila Pa 1976) 1996;21:941-4.

7. Motosuneya T, Asazuma T, Tsuji T, Watanabe H, Nakayama Y, Nemoto K. Postoperative change of the cross-sectional area of back musculature after 5 surgical procedures as assessed by magnetic resonance imaging. J Spinal Disord Tech 2006;19:318-22.

8. Kim KT, Lee SH, Suk KS, Bae SC. The quantitative analysis of tissue injury markers after mini-open lumbar fusion. Spine (Phila Pa 1976) 2006;31:712-6.

9. Ward SR, Kim CW, Eng CM, et al. Architectural analysis and intraoperative measurements demonstrate the unique design of the multifidus muscle for lumbar spine stability. J Bone Joint Surg Am 2009;91:17685.

10. Bogduk N, Wilson AS, Tynan W. The human lumbar dorsal rami. J Anat 1982;134(Pt 2):383-97.

11. Kawaguchi Y, Matsui H, Gejo R, Tsuji H. Preventive measures of back muscle injury after posterior lumbar spine surgery in rats. Spine (Phila Pa 1976) 1998;23:2282-7. 
12. Suwa H, Hanakita J, Ohshita N, Gotoh K, Matsuoka $\mathrm{N}$, Morizane A. Postoperative changes in paraspinal muscle thickness after various lumbar back surgery procedures. Neurol Med Chir (Tokyo) 2000;40:151-4.

13. Kim K, Isu T, Sugawara A, Matsumoto R, Isobe M. Comparison of the effect of 3 different approaches to the lumbar spinal canal on postoperative paraspinal muscle damage. Surg Neurol 2008;69:109-13.

14. Remes V, Lamberg $\mathrm{T}$, Tervahartiala $\mathrm{P}$, et al. Longterm outcome after posterolateral, anterior, and circumferential fusion for high-grade isthmic spondylolisthesis in children and adolescents: magnetic resonance imaging findings after average of 17-year follow-up. Spine (Phila Pa 1976) 2006;31:2491-9.

15. Gillet $P$. The fate of the adjacent motion segments after lumbar fusion. J Spinal Disord Tech 2003;16:33845.

16. Helgeson MD, Bevevino AJ, Hilibrand AS. Update on the evidence for adjacent segment degeneration and disease. Spine J 2013;13:342-51.

17. Datta G, Gnanalingham KK, Peterson D, et al. Back pain and disability after lumbar laminectomy: is there a relationship to muscle retraction? Neurosurgery 2004;54:1413-20.

18. Styf JR, Willen J. The effects of external compression by three different retractors on pressure in the erector spine muscles during and after posterior lumbar spine surgery in humans. Spine (Phila Pa 1976) 1998;23:354-8.

19. Pradhan BB, Nassar JA, Delamarter RB, Wang JC. Single-level lumbar spine fusion: a comparison of anterior and posterior approaches. J Spinal Disord Tech 2002;15:355-61.
20. Cholewicki J, Panjabi MM, Khachatryan A. Stabilizing function of trunk flexor-extensor muscles around a neutral spine posture. Spine (Phila Pa 1976) 1997;22:2207-12.

21. Fan S, Hu Z, Zhao F, Zhao X, Huang Y, Fang X. Multifidus muscle changes and clinical effects of onelevel posterior lumbar interbody fusion: minimally invasive procedure versus conventional open approach. Eur Spine J 2010;19:316-24.

22. Moon KY, Lee SE, Kim KJ, Hyun SJ, Kim HJ, Jahng TA. Back muscle changes after pedicle based dynamic stabilization. J Korean Neurosurg Soc 2013;53:1749.

23. Hyun SJ, Kim YB, Kim YS, et al. Postoperative changes in paraspinal muscle volume: comparison between paramedian interfascial and midline approaches for lumbar fusion. J Korean Med Sci 2007;22:646-51.

24. Hu Y, Leung HB, Lu WW, Luk KD. Histologic and electrophysiological changes of the paraspinal muscle after spinal fusion: an experimental study. Spine (Phila Pa 1976) 2008;33:1418-22.

25. Cawley DT, Alexander M, Morris S. Multifidus innervation and muscle assessment post-spinal surgery. Eur Spine J 2014;23:320-7.

26. Yoo JS, Min SH, Yoon SH, Hwang CH. Paraspinal muscle changes of unilateral multilevel minimally invasive transforaminal interbody fusion. J Orthop Surg Res 2014;9:130. 